\title{
Perfil de fuerza isométrica máxima de los rotadores de la cadera de un equipo senior de fútbol
}

\author{
Isometric maximum strength profile of hip rotators
} of a senior soccer team

\begin{abstract}
Antonio Cejudo'
José Manuel Armada-Zarco'

Riccardo $\mid z z O^{2}$

Pilar Sainz de Baranda'

1. Departamento de Actividad Física y Deportiva, Facultad de Ciencias del Deporte, Campus de Excelencia Internacional de la Universidad de Murcia "Campus Mare Nostrum", Universidad de Murcia, Murcia, España.

2. Dipartimento di Scienze Biomolecolari, Scuola di Scienze Motorie, Università degli Studi, Urbino, Italy.
\end{abstract}

\begin{abstract}
Resumen
La debilidad y desequilibrios de los rotadores de cadera están asociados con las lesiones en los deportistas. El objetivo del presente estudio fue describir el perfil de fuerza isométrica máxima (FIM) de los rotadores internos (RIC) y externos (REC) de la cadera en jugadores senior de fútbol. Para ello, se realizó un estudio observacional, descriptivo y de corte transversal con 21 jugadores senior de fútbol que participaron voluntariamente en esta experiencia. La FIM de la RIC y REC se midió con un dinamómetro manual Lafayette. Se realizó una prueba t de Student para determinar la existencia de posibles diferencias entre la FIM de la cadera dominante y no dominante, y entre los valores de la FIM de RIC y REC. Estos análisis se complementaron con el cálculo del tamaño del efecto mediante la d de Cohen. Los jugadores mostraron resultados de FIM de 200,94 $\pm 29,98 \mathrm{~N}, 2,66 \pm 0,37 \mathrm{~N} / \mathrm{kg}$ de masa corporal y 1,09 $\pm 0,16 \mathrm{~N} * \mathrm{~m} / \mathrm{kg}$ de masa corporal en la REC; y 154,22 $\pm 25,09 \mathrm{~N}, 2,04 \pm 0,33 \mathrm{~N} / \mathrm{kg}$ de masa corporal y 0,84 $\pm 0,14 \mathrm{~N} * \mathrm{~m} / \mathrm{kg}$ de masa corporal en la RIC. El análisis comparativo de la FIM en las diferentes unidades de medida mostró valores superiores en los REC que en los RIC ( $p=0,000 ; d$ de Cohen > 1,631 [grande]). Tomando como base podemos concluir, que el procedimiento estandarizado de evaluación empleado en este estudio ha obtenido valores normativos del perfil de FIM de los rotadores de la cadera superiores a los descritos para otros jugadores de fútbol de nivel competitivo superior de ambos sexos. Los valores de la FIM de los REC son superiores a los RIC.
\end{abstract}

Palabras clave: Dinamómetro manual, deportes colectivos, test de campo, valores de referencia, desequilibrio muscular, lesión por sobreuso.

\begin{abstract}
Hip rotator weakness and imbalances are associated with injuries in athletes. The aim of the present study was to describe the maximal isometric strength (MIS) profile of the internal (HIR) and external (HER) hip rotators in senior soccer players. An observational, descriptive, cross-sectional study was conducted in 21 senior soccer players that voluntarily participated in the present study. The MIS of HIR and HER were measured with the use of a manual Lafayette dynamometer. A Student's t-test for related data (dependent samples) was calculated to determine the existence of differences between the MIS values of the dominant and nondominant hip, and between the MIS values of the HIR and HER. These analyses were complemented with the effect size of all outcomes using Cohen's d statistic. The players showed MIS results of $200.94 \pm 29.98 \mathrm{~N}, 2.66 \pm 0.37 \mathrm{~N} / \mathrm{kg}$ body mass and $1.09 \pm 0.16 \mathrm{~N} * \mathrm{~m} / \mathrm{kg}$ body mass in the REC; and $154.22 \pm 25.09$ $\mathrm{N}, 2.04 \pm 0.33 \mathrm{~N} / \mathrm{kg}$ body mass and $0.84 \pm 0.14 \mathrm{~N} * \mathrm{~m} / \mathrm{kg}$ body mass in the RIC. Comparison analysis of MIS means in the different units of measurement showed higher values in HER than in HIR ( $p=0.000$; Cohen's $d>1.631$ [large]). In conclusion, a standardized assessment method has obtained normative values of the FIM profile of hip rotators higher than other soccer players of higher competitive level and both sexes. The FIM values of HER are higher than HIR.
\end{abstract}

Keywords: Hand-held dynamometer, team sports, field test, reference values, muscle imbalance, overuse injury.

*Autor de correspondencia: Antonio Cejudo, antonio.cejudo@um.es

Recibido: 10 de junio de 2021

Aceptado: 21 de julio de 2021

Publicado: 01 de diciembre de 2021

Como citar (APA): Cejudo, A., Armada-Zarco, J. M., Izzo, R., \& Sainz de Baranda, P. (2021). Perfil de fuerza isométrica máxima de los rotadores de la cadera de un equipo senior de fútbol. JUMP, (4), 1-9. https://doi.org/10.17561/jump.n4.1

This is an open access article under the CC-BY 4.0 license

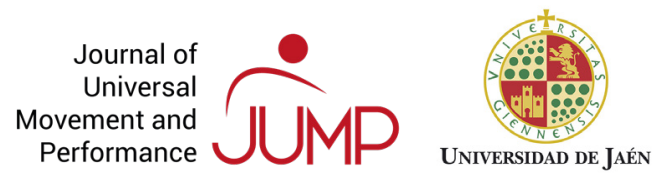




\section{Introducción}

La articulación de la cadera está diseñada para proporcionar estabilidad corporal en sacrificio del movimiento (Clarkson, 2003; Palmer \& Epler, 2002). Para el desempeño de esta función, los rotadores de la cadera se consideran los músculos más importantes por sus funciones de estabilización del tronco, la cadera y rodilla (Clarkson, 2003; Kapandji, 2007). Sin embargo, se requiere cierto equilibrio de fuerza entre los rotadores externos (REC) e internos (RIC) de la cadera debido a ciertas diferencias anatómicas. Los REC son más numerosos y potentes que sus antagonistas (Kapandji, 2007); éstos son los pelvitrocantéreos (piramidal, el obturador interno, el géminos pélvico y el obturador externo), los glúteos (mayor, mediano y menor) y algunos aductores (cuadrado crural, pectíneo y aductor mayor) que actúan en determinadas ocasiones en esta rotación. Mientras que los RIC (tensor de la fascia lata, el glúteo menor y mediano) son menos numerosos y tres veces menos potentes que sus antagonistas (Kapandji, 2007).

Cada vez hay más estudios científicos que han encontrado una asociación causal entre la debilidad de los rotadores de la cadera y las desalineaciones del miembro inferior como el signo de Trendelenburg (Brukner et al., 2014), el valgo de rodilla (Dierks et al., 2008; Wilczyński et al., 2021) y la torsión femoral (Hafiz, 2014) en la población general y deportista. También, este factor de riesgo se ha asociado con lesiones de la rodilla como el síndrome femoropatelar (Dierks et al., 2008; Powers, 2010) y la rotura del ligamento cruzado anterior (Powers, 2010; Sigward et al., 2008) en deportistas. Los desequilibrios musculares unilaterales (agonistas versus antagonista) y bilaterales (rotadores de la cadera externos más fuertes que los internos o viceversa) también han sido establecidos como factores de riesgo de diferentes problemas músculoesqueléticos en deportistas (Cibulka et al., 2010) como el dolor lumbar (Ellison et al., 1990; Hides \& Oostenbroek, 2016), el dolor patelofemoral (Cibulka \& Threlkeld-Watkins, 2005; Leporace et al., 2018) y el esguince de tobillo (De Ridder et al., 2017). Además, se ha demostrado que ambos factores de riesgo de lesión, debilidad y desequilibrio muscular, causan disminución del rendimiento físico-técnico deportivo en acciones técnicas tales como el golpeo de balón (Lees et al., 2010), el cambio de dirección, la deceleración (Howard et al., 2011), el aterrizaje tras el salto (Howard et al., 2011) o el sprint (Ocarino et al., 2021).

La evaluación de la fuerza isométrica máxima (FIM) de los rotadores de la cadera puede proporcionar información interesante para la gestión de estas lesiones deportivas en términos de prevención y readaptación físico-técnica. En el ámbito deportivo, clínico y de investigación el uso del dinamómetro manual es frecuente por su accesibilidad, coste económico, portabilidad y fácil uso (De Ridder et al., 2017; Desmyttere et al., 2019; Hannon et al., 2019; Leporace et al., 2018; Ocarino et al., 2021). Además, se ha demostrado la validez y fiabilidad de la medición de la FIM con este instrumento en adultos activos (Thorborg et al., 2013) y futbolistas (Desmyttere et al., 2019; Paul \& Nassis, 2015). Sin embargo, los estudios que han evaluado la FIM en los deportistas han usado diferentes metodologías y marcas de instrumentos de medición (De Ridder et al., 2017; Desmyttere et al., 2019; Hannon et al., 2019; Hides \& Oostenbroek, 2016; Leporace et al., 2018; Ocarino et al., 2021; Sigward et al., 2008). Este hecho, puede justificar los resultados discrepantes de FIM observados en la población deportista (Baldon et al., 2012; Ocarino et al., 2021). Así, la aplicación adicional o no de la fuerza aplicada por el evaluador al retener el empuje del deportista (De Ridder et al., 2017; Hannon et al., 2019; Hides \& Oostenbroek, 2016; Leporace et al., 2018; Sigward et al., 2008), el control o no de los movimientos compensatorios (Jackson et al., 2017; Ocarino et al., 2021; Romero-Franco et al., 2017; Sigward et al., 2008), la postura del explorado en decúbito supino, prono o lateral (Baldon et al., 2012), y la posición de la flexión de cadera neutra versus flexionada (Baldon et al., 2012) son variables procedimentales que pueden afectar a los resultados de FIM.

Para identificar el riesgo de lesión deportiva asociada a la debilidad y desequilibrio muscular, los profesionales de las Ciencias del Deporte deben de disponer de valores de referencia normativos de la FIM obtenidos mediante una metodología estandarizada que evite la variabilidad en los datos. Por ello, el objetivo del presente estudio fue describir y analizar los valores normativos del perfil de FIM de los REC y 
RIC en jugadores senior de fútbol. Las hipótesis del estudio fueron: a) los valores de FIM de los jugadores profesionales de fútbol son superiores a aquellos con nivel competitivo regional; b) los valores de RIC son superiores a aquellos obtenidos por los REC en los jugadores senior de fútbol.

\section{Método}

\section{Diseño de la investigación}

Se realizó un estudio observacional, descriptivo y de corte transversal con 21 jugadores masculinos senior de fútbol. Tras informar sobre los propósitos del estudio a la dirección y staff técnico del club durante la pretemporada, los jugadores fueron reclutados antes de la primera competición oficial.

La última semana de la pretemporada se registraron los datos demográficos, antropométricos y experiencia deportiva en un cuestionario estandarizado. Los evaluadores tomaron los datos antropométricos y aclararon las posibles dudas sobre el cuestionario. Los jugadores se familiarizaron con el procedimiento de evaluación de la FIM durante la misma semana. Por indicación del entrenador del equipo, la FIM de los REC y RIC se midió en todos los jugadores la primera semana del periodo competitivo y antes del inicio de la sesión de recuperación. La sesión de evaluación se realizó en la habitación de fisioterapia del club. La FIM se midió en decúbito prono con la cadera neutra y la rodilla flexionada a $90^{\circ}$. Antes de la sesión de medición, un calentamiento específico dirigido por un educador físico-deportivo fue aplicado a todos los jugadores. Los jugadores fueron evaluados con la vestimenta habitual de entrenamiento. El orden de los test de evaluación de la FIM fue aleatorio. Los dos evaluadores aprendieron y perfeccionaron el procedimiento de medición de la FIM durante las dos temporadas deportivas previas al inicio de esta investigación. Tres repeticiones fueron realizadas de cada cadera en ambos movimientos, y su promedio fue utilizado para el posterior análisis estadístico (Ocarino et al., 2021; Vannatta \& Kernozek, 2021). Si los evaluadores observaron una diferencia superior al $5 \%$ en los datos obtenidos, una cuarta o quinta repetición fue realizada. La media de las tres medidas más próximas fue usada para el posterior análisis estadístico. Al finalizar el periodo competitivo, los jugadores completaron un cuestionario sobre su historial deportivo con la ayuda de los evaluadores y staff técnico del club.

\section{Participantes}

La muestra estuvo compuesta por 21 jugadores masculinos senior de un equipo de fútbol que competían en el Grupo B de Preferente Autonómica de una liga regional. Todos los jugadores participaron voluntariamente en el estudio. Los jugadores presentaron una media de $23,33 \pm 3,01$ años de edad, $75,73 \pm 7,10 \mathrm{~kg}$ de masa corporal, $177,90 \pm 5,53 \mathrm{~cm}$ de altura corporal y $23,91 \pm 1,75 \mathrm{~kg} / \mathrm{m}^{2}$ de índice de masa corporal. Los jugadores tenían una media de $14,95 \pm 3,86$ años de experiencia competitiva federada; en la temporada deportiva anterior promediaron $3,05 \pm 0,21$ días de entrenamiento semanal, 4,57 $\pm 0,32$ horas de entrenamiento por semana y $65,71 \pm 30,34$ minutos de participación en competición.

Los jugadores no mostraron problemas ortopédicos en la extremidad inferior o la columna vertebral que pudieran afectar a la competencia físico-técnicas deportivas, a las características antropométricas y/o a la FIM de la cadera durante las dos semanas previas a la sesión de evaluación. Además, se excluyeron los porteros y a aquellos jugadores de campo que mostraron agujetas en la sesión de evaluación por las competiciones de pretemporada.

El estudio fue diseñado siguiendo las directrices establecidas en la Declaración de Helsinki. El protocolo de evaluación de la FIM fue aprobado por el Comité de Ética e Investigación de la Universidad de Murcia (ID: 1672/2017). Antes del inicio del estudio, los jugadores confirmaron que habían leído la información relacionada con la investigación y firmaron el consentimiento informado para su participación. Los jugadores fueron informados de la opción de retirarse del estudio de forma voluntaria sin dar explicaciones de ningún tipo. Además, antes y durante el estudio, los evaluadores respondieron a todas las preguntas realizadas por los jugadores.

\section{Evaluadores}

La toma de datos de este estudio fue realizada por dos evaluadores. Ambos eran graduados en 
Ciencias de la Actividad Física y el Deporte (CAFD) y presentaban al menos 2 años de experiencia en la evaluación de la FIM. Cada evaluador presentaba unas competencias específicas para el desarrollo de los test, que fueron mantenidas en todos los jugadores. El evaluador principal colocaba el instrumento de medición y medía la fuerza, y el evaluador asistente evitaba los posibles movimientos compensatorios. Un ayudante familiarizado con el procedimiento registraba el dato en la planilla de registro.

En un estudio previo a doble-ciego de dos sesiones de medición separadas por 24 horas con 10 adultos jóvenes activos, los evaluadores demostraron una fiabilidad intraevaluador de las medidas excelente, con un coeficiente de correlación intraclase superior a 0,89 (REC= 0,90-0,98; $\mathrm{RIC}=0,89-0,96)$ y un error estándar de la media inferior o igual a 10,8 N (REC=8,6N; $\mathrm{RIC}=10,8 \mathrm{~N}$ ) para las variables de FIM medidas.

\section{Test de evaluación de la fuerza isométrica} máxima

En la sesión de familiarización, los jugadores recibieron una explicación completa de los procedimientos de evaluación. Antes de las mediciones de FIM, los jugadores corrieron cinco minutos alrededor del campo de fútbol a una intensidad de $8 \mathrm{~km} * \mathrm{~h}^{-1}$ y ejecutaron dos repeticiones progresivas hasta el $80 \%$ de FIM como calentamiento siguiendo las directrices de dos estudios previos (Dierks et al., 2008; Widler et al., 2009).

La FIM de los RIC (Figura 1a) y REC (Figura 1b) se midieron en decúbito prono con la cadera neutra $\mathrm{O}$ a $0^{\circ}$ y la rodilla flexionada a $90^{\circ}$. El dinamómetro se localizó en la parte distal de la pierna a $5 \mathrm{~cm}$ del maléolo peroneal del tobillo para los RIC y del maléolo tibial para los REC (Sigward et al., 2008; Thorborg et al., 2010). Para los REC se utilizó un dispositivo o brazo extensible que permitió el contacto del instrumento de medida con la pared (Figura 1b). A cada jugador se le pidió la aplicación de la FIM contra la pared durante 5 segundos (Ocarino et al., 2021; Sigward et al., 2008). De esta forma, la medida obtenida no estuvo influenciada por la fuerza adicional realizada por el evaluador al intentar retener el movimiento (Magalhães et al., 2013; Sigward et al., 2008). El evaluador principal animó verbalmente a los jugadores durante el procedimiento con la frase "adelanteempuja-empuja-empuja y relájate" para obtener la aplicación de fuerza máxima. Entre las repeticiones y los test los jugadores realizaron un descanso de 60 segundos. Se empleó un dinamómetro manual Lafayette (Lafayette ${ }^{\circledR}$ Instrument Company, Lafayette, Indiana) para medir la FIM en Newtons. Para normalizar esta medida, el resultado de la FIM fue dividido por la masa corporal (Sigward et al., 2008). La estimación del momento del brazo de palanca o torque máximo se calculó multiplicando el valor de la FIM (Newtons) por la longitud de la tibia (metros), y posteriormente, el resultado se dividió por la masa corporal (Sigward et al., 2008). El control de los movimientos compensatorios fue competencia específica del evaluador asistente dando instrucciones correspondientes al jugador evaluado cuando fue necesario. En la evaluación de los RIC se evitó la elevación de la hemipelvis contralateral y los movimientos como la abducción o extensión de cadera; mientras que en la evaluación de los REC se impidió la elevación de la hemipelvis homolateral y los movimientos de cadera como la aducción o extensión. El test fue repetido si el evaluador asistente no podía controlar los movimientos compensatorios (Ocarino et al., 2021; Sigward et al., 2008).
(a) Rotación interna (b) Rotación externa

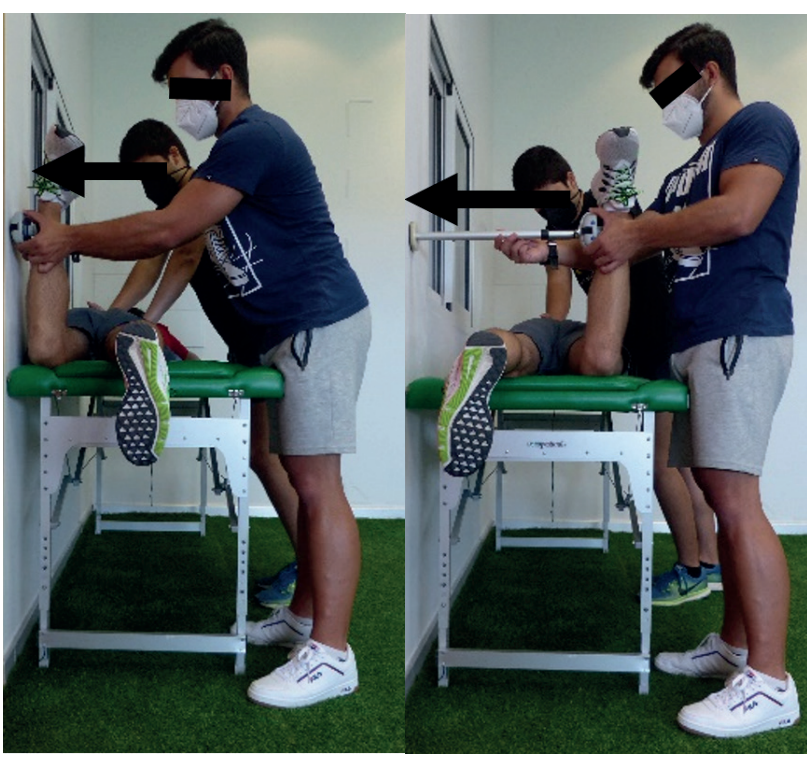

Figura 1. Evaluación de la fuerza isométrica máxima de los rotadores de la cadera. 


\section{Análisis estadístico}

Previo al análisis estadístico, se aplicó el test Shapiro-Wilk y el test de homocedasticidad a las variables continuas para determinar o no la distribución normal de los datos. Un análisis de potencia post hoc fue realizado para calcular la potencia del tamaño muestral a posteriori, siguiendo una metodología descrita previamente (Faul et al., 2007) y empleando el programa informático G*Power 3.1.9.7.

Los datos de las variables medidas (edad, antropometría, experiencia deportiva y FIM) fueron analizados usando estadísticos descriptivos y se expresan como media y su correspondiente desviación típica.

Una prueba $t$ de Student para datos relacionados (muestras dependientes) se aplicó para determinar la existencia de diferencias entre la FIM de la cadera dominante y no dominante, y entre los valores de FIM para los RIC y REC. Estos análisis fueron complementados con el tamaño del efecto mediante el estadístico d de Cohen. Este dato fue interpretado cualitativamente con la clasificación propuesta por Hopkins (Hopkins et al., 2009) de la siguiente manera: extremadamente grande $(>4,0)$, muy grande $(2,00$ a 3,99$)$, grande $(1,20$ a 2,00$)$, moderado $(0,6$ a 1,19$)$, pequeño $(0,2$ a 0,59$)$ o trivial $(<$ 0,2 ). Los autores consideraron arbitrariamente "moderado" como el nivel mínimo de tamaño de efecto relevante con aplicación práctica de los resultados (Hopkins, 2004). Un p-valor inferior a 0,05 fue establecido como nivel de significancia estadística $(p<0,05)$.

\section{Resultados}

Tras añadir los parámetros de entrada, el tamaño del efecto ( $d$ Cohen; diferencias de dos muestras dependientes -FIM REC vs RIC-), el tamaño muestral del estudio $(n=21)$ y la probabilidad de error $(a=0,05)$, en el software $G *$ Power 3.1.9.4 fue obtenida una potencia estadística de 1,000 (1- $\beta$ error prob).

No se encontraron diferencias significativas entre el lado dominante y no dominante para los RIC (dominante: 154,37 $\pm 28,26 \mathrm{~N}$ versus No dominante: $154,04 \pm 25,51 \mathrm{~N} ; \mathrm{p}=0,938$; $d$ de Cohen: 0.000 -Trivial-) y los REC (dominante: $204,43 \pm 32,59 \mathrm{~N}$ versus No dominante: 197,41 $\pm 30,33 \mathrm{~N} ; \mathrm{p}=$ 0,109; $d$ de Cohen: 0,223 -Trivial-). Por tanto, la media de ambos lados corporales fue usada para describir el perfil de FIM de los rotadores de cadera en valores absolutos (Newtons), normalizados con la masa corporal (Newtons/kg de masa corporal) y torque (Newtons*metro/kg de masa corporal-) en los jugadores senior de fútbol (Tabla 1).

Se observaron diferencias significativas entre los REC y RIC ( $p=0,000$; d de Cohen > 1,631 [grande]) en las medias de FIM (Tabla 1).

Tabla 1. Resultados de la fuerza isométrica máxima de los rotadores de la cadera en jugadores senior de fútbol

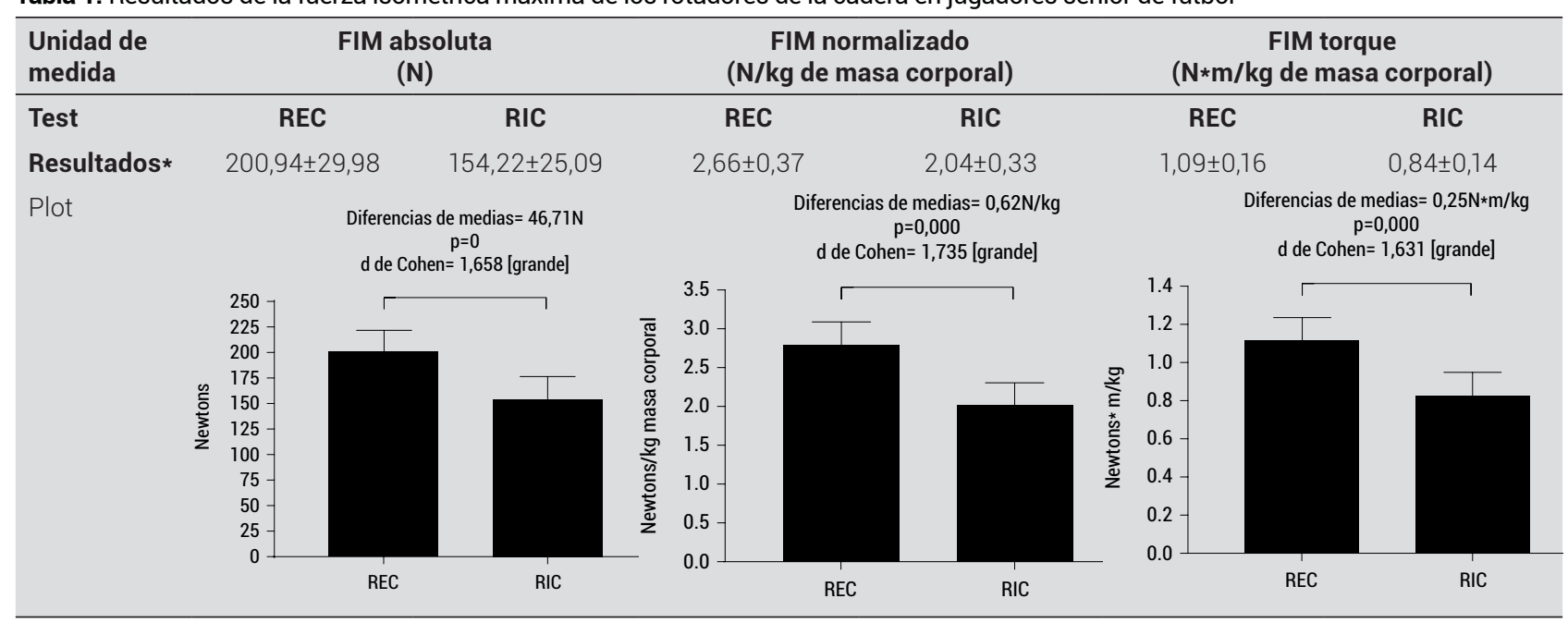

Nota *: Media \pm desviación estándar; FIM: fuerza isométrica máxima; N: Newtons; RIC: rotadores internos de la cadera; REC: rotadores externos de la cadera. 


\section{Discusión}

El proceso de identificación de los jugadores con riesgo de lesión deportiva precisa de valores de referencia normativos obtenidos con un procedimiento de evaluación válido y preciso (Hayen et al., 2007; Hopkins et al., 2009). En este sentido, el presente estudio es, bajo el conocimiento de los autores, el primer trabajo que describe el perfil de FIM de los rotadores de la cadera con un procedimiento de evaluación estandarizado que evita repetir los principales errores metodológicos observados en estudios previos. En este sentido, los evaluadores han prestado un gran interés en el control de los posibles movimientos compensatorios, específicamente mediante la asignación de competencias específicas sobre este aspecto al evaluador asistente y la transmisión de instrucciones específicas a los jugadores; además, los jugadores siempre han aplicado su fuerza máxima contra una de las paredes de la habitación de evaluación para conseguir una contracción isométrica pura (De Ridder et al., 2017; Hannon et al., 2019; Hides \& Oostenbroek, 2016; Leporace et al., 2018; Sigward et al., 2008). Si las compensaciones no se controlan o el evaluador retiene la fuerza del jugador con sus manos, la suma de fuerza de los músculos sinergistas u otros músculos del cuerpo y la fuerza aplicada por el evaluador, respectivamente, van a proporcionar una fuerza extra o un resultado falso positivo. Los estudios que han medido la FIM de los rotadores de la cadera en jugadores de fútbol han evaluado en tres posiciones corporales como son la sedestación con la cadera en flexión de $90^{\circ}$ (Cichanowski et al., 2007; De Ridder et al., 2017; Sigward et al., 2008), en decúbito prono con la cadera a $0^{\circ}$ (Hannon et al., 2019; Leporace et al., 2018; Ocarino et al., 2021), y en decúbito supino con la cadera a $0^{\circ}$ (Hides \& Oostenbroek, 2016) y a $90^{\circ}$ (Desmyttere et al., 2019). Diferentes dinamómetros manuales como las marcas Lafayette ${ }^{\circledR}$ (Leporace et al., 2018; Magalhães et al., 2013), MicroFET2 ${ }^{\circledR}$ han sido usados para medir la FIM (Cibulka et al., 2010; Cichanowski et al., 2007; De Ridder et al., 2017; Ocarino et al., 2021; Vannatta \& Kernozek, 2021). Los valores de la FIM han sido reportados por los autores en diferentes unidades de medida como Newtons (De Ridder et al., 2017), Newtons* longitud de la tibia (Desmyttere et al., 2019; Hides \& Oostenbroek, 2016), Newtons/kg de masa corporal (Hannon et al., 2019) y torque -Newtow * longitud de la tibia/kg de masa corporal- (Desmyttere et al., 2019; Ocarino et al., 2021; Sigward et al., 2008). Con estos antecedentes metodológicos, y con el fin de facilitar la comparación con los estudios publicados previamente, los resultados del presente estudio han sido presentados en las tres unidades de medidas empleadas en las investigaciones citadas.

Tras no observar diferencias significativas entre la FIM de los rotadores de la cadera dominante y la cadera no dominante, el promedio de los valores de ambos lados corporales se usó para establecer el perfil FIM de los rotadores de la cadera. Los resultados del perfil de FIM de los REC (Newtons, Newtons/kg de masa corporal y torque) de este estudio medido en decúbito prono son superiores a aquellos publicados en 317 jugadores profesionales de fútbol (Leporace et al., 2018; Ocarino et al., 2021). Los valores inferiores de ambos estudios pueden ser causados por la menor longitud de la tibia al colocar el instrumento de medida a $15 \mathrm{~cm}$ del calcáneo (Ocarino et al., 2021) y la mayor masa corporal de los jugadores profesionales de fútbol (Leporace et al., 2018). El rendimiento en el test de FIM puede estar también afectado por la ausencia de la sesión de familiarización del test (Leporace et al., 2018) o de un calentamiento pretest (Ocarino et al., 2021). Así como, la aplicación de una única repetición de fuerza isométrica submáxima, lo que puede ser insuficiente para lograr un rendimiento óptimo en el test (Leporace et al., 2018). Estudios previos han demostrado que la familiarización (Lange Chamorro, 2017; Vega Cerda, 2018) y el calentamiento (Siff \& Verkhoshansky, 2004; Vega Cerda, 2018) influyen positivamente en el rendimiento de diferentes test de fuerza. Por último, la carga de entrenamiento de la fuerza de los REC también puede influir en los resultados (Lange Chamorro, 2017; Siff \& Verkhoshansky, 2004).

En 30 jugadoras de fútbol adolescentes, Hannon et al. (Hannon et al., 2019) también encontraron valores inferiores de FIM a los resultados de este estudio. Estas diferencias pueden ser explicadas por el diferente nivel 
competitivo y la edad entre ambas muestras (Ocarino et al., 2021), y el sexo (Lindsay et al., 1992; Thorborg et al., 2013; Vannatta \& Kernozek, 2021). En este estudio (Hannon et al., 2019), si incluyeron un calentamiento de 10 minutos y 5 repeticiones submáximas de familiarización previamente a la realización de los test de FIM. Sin embargo, la retención de la fuerza aplicada por las jugadoras y el agarre del dinamómetro manual por el evaluador puede haber afectado a los resultados (Hannon et al., 2019; Leporace et al., 2018).

Otros autores han evaluado la FIM en sedestación con la cadera flexionada a $90^{\circ}$ en 133 jugadores jóvenes de fútbol (De Ridder et al., 2017) y en 31 jugadoras de fútbol universitarias (Sigward etal., 2008). Enambos estudiostambién se obtuvieron valores de FIM de los rotadores de la cadera inferiores. Estos resultados pueden ser lógicos porque con la cadera a $0^{\circ}$ (posición en decúbito prono) hay un mayor número de músculos con brazo de momento rotacional externo que con la cadera en flexión a $90^{\circ}$ como en sedestación (Baldon et al., 2012; Kapandji, 2007). Además, Kapadji (Kapandji, 2007) muestra diferencias entre el número y el tamaño de los músculos rotadores de la cadera en ambas posiciones (cadera flexionada a $0^{\circ}$ y a $90^{\circ}$ ). El bíceps femoral, el semitendinoso, el semimembranoso, aductor mediano y aductor menor actúan como sinergistas con la cadera neutra. El piramidal y glúteos -menor y mediano- actúan como sinergistas de los rotadores de cadera con la flexión de cadera a $90^{\circ}$ (Kapandji, 2007). Estos argumentos anatómicos pueden justificar las diferencias de resultados entre ambas posiciones y entre las diferentes muestras de jugadores de fútbol de ambos estudios (De Ridder et al., 2017; Sigward et al., 2008). Asimismo, la edad de los jugadores, la ausencia de una sesión de familiarización y un calentamiento previo a la ejecución de los test del estudio de De Ridder et al. (2017) pueden explicar las diferencias de resultados. Mientras que en el estudio de Sigward et al. (Sigward et al., 2008) puede influir las diferentes características antropométricas (Vannatta \& Kernozek, 2021) y físicas (Meylan et al., 2008) relacionadas con el sexo de la muestra como ha sido demostrado en estudio previos (Meylan et al., 2008; Vannatta \& Kernozek, 2021). Por último, los valores inferiores de torque en los REC observados en estos estudios (De Ridder et al., 2017; Sigward et al., 2008) pueden ser explicados por los valores inferiores de FIM absoluta obtenidos y la menor altura corporal de las jugadoras de fútbol.

En base a las discrepancias metodológicas mencionadas anteriormente y posiciones de evaluación, estos resultados deben interpretarse con cautela. Como ha sido descrito en el presente estudio, la evaluación de la FIM con dinamómetro manual requiere de un procedimiento estandarizado que incluya una sesión de familiarización de los tests, la aplicación de un calentamiento normalizado, el control exhaustivo de movimientos compensatorios (cinchas, examinador asistente o ambos), el uso instrumento de medida por el evaluador que obtenga una FIM pura, la medición, el tiempo de descanso y el promedio de tres o más repeticiones para determinar los valores normativos de la muestra.

Para obtener los valores normativos de la FIM de los rotadores de la cadera, futuros estudios deben de reclutar una muestra representativa de este nivel competitivo y sexo. La FIM debe ser medida después de la sesión de recuperación para evitar la influencia de la fatiga o las agujetas post-competición sobre el rendimiento de los test de FIM. Así como, sería interesante controlar la carga de entrenamiento de la fuerza de los rotadores de la cadera, específicamente los REC, para profundizar en la discusión de los resultados.

\section{Conclusiones}

El procedimiento estandarizado de evaluación empleado en este estudio, ha obtenido valores normativos del perfil de FIM de los rotadores de la cadera superiores a los descritos para jugadores de fútbol de nivel competitivo superior y ambos sexos. Los valores de la FIM de los REC son superiores a los RIC.

\section{Aplicaciones prácticas}

El modelo de intervención para el rendimiento óptimo con un menor riesgo de lesión consecuente de este estudio puede basarse en las siguientes competencias profesionales: 
1. Entrevistar al jugador con la ayuda de un cuestionario que recopile la información del historial deportivo y lesional.

2. Evaluar la FIM mediante un procedimiento estandarizado, que proporcione unos datos válidos y fiables. Este procedimiento debe incluir una sesión previa de familiarización de los test, la aplicación de un calentamiento general y un calentamiento específico con la práctica del test con una carga submáxima, la evaluación en decúbito prono por la estabilidad-comodidad del jugador y el control de movimientos compensatorios, el control de movimientos compensatorios por un evaluador asistente e instrucciones específicasaljugador evitando laineficacia de las cinchas, la repetición de test hasta conseguir tres medidas similares por la variabilidad de la medida causada por la complejidad de la ejecución del test y un intervalo de tiempo suficiente que evite la acumulación de fatiga muscular (al menos 60 segundos).

3. Normalizar los valores de FIM con la masa corporal. Además, el cálculo biomecánico del torque teniendo en cuenta la longitud de la tibia y la masa corporal sería un dato muy interesante.

4. Comparar los resultados del jugador con los valores normativos o de referencia de la FIM de los rotadores de la cadera del fútbol federado. Los valores normativos del presente estudio deben de tratarse con cautela por el insuficiente tamaño muestral para generalizar los resultados a los jugadores de fútbol. Los valores normativos proporcionan a los preparadores y readaptadores físicos los objetivos de entrenamiento de la fuerza de los rotadores de cadera para incrementar el rendimiento físico-técnico con un menor riesgo de lesión deportiva. Estos valores deben ser específicos del deporte, el nivel competitivo y el sexo.

5. Aplicar un programa de entrenamiento de fuerza si el jugador muestra valores inferiores a los valores de normativos. En términos de transferencia físico técnica deportiva, se sugiere la activación de esta musculatura durante las acciones técnicas individuales y colectivas del fútbol que favorezcan las desalineaciones de la región lumbopélvica o del miembro inferior como el valgo de rodilla, ángulo Q o disfunción femororrotuliana, o signo de Trendelenburg. Así como, la calidad de ejecución de estas acciones técnicas propios del fútbol que soliciten los rotadores de la cadera como el chut, la conducción, el regate o el cambio de dirección.

\section{Financiación}

Este estudio es parte del Proyecto de Investigación financiado por FEDER/ Ministerio de Ciencia, Innovación y Universidades - Agencia Estatal de Investigación/ Proyecto "Estudio del riesgo de lesión en jóvenes deportistas a través de redes de inteligencia artificial" (DEP201788775-P).

\section{Referencias}

Baldon, R., Furlan, L., \& Serrão, F. (2012). Influence of the hip flexion angle on isokinetic hip rotator torque and acceleration time of the hip rotator muscles. Journal of Applied Biomechanics, 29(5), 593-599. https://doi.org/10.1123/jab.29.5.593

Brukner, P., Nealon, A., Morgan, C., \& Burgess, D. (2014). Recurrent hamstring muscle injury: applying the limited evidence in the professional football setting with a seven-point programme. British Journal of Sports Medicineournal of Sports Medicine, 48(11), 929-938.

https://doi.org/10.1136/bjsports-2012-091400

Cibulka, M., \& Threlkeld-Watkins, J. (2005). Patellofemoral Pain and Asymmetrical Hip Rotation. Physical Therapy, 85(11), 12011207. https://doi.org/10.1093/PTJ/85.11.1201

Cibulka, M., Strube, M., Meier, D., Selsor, M., Wheatley, C., Wilson, N., \& Irrgang, J. (2010). Symmetrical and asymmetrical hip rotation and its relationship to hip rotator muscle strength. Clinical Biomechanics, 25(1), 56-62 https://doi.org/10.1016/j.clinbiomech.2009.09.006

Cichanowski, H., Schmitt, J., Johnson, R., Niemuth, P., Schmitt, J., Johnson, R., \& Niemuth, E. (2007). Hip Strength in Collegiate Female Athletes with Patellofemoral Pain. Medicine \& Science in Sports \& Exercise, 39(8), 1227-1232.

https://doi.org/10.1249/mss.0b013e3180601109

Clarkson, H. (2003). Proceso evaluativo musculoesquelético: amplitud del movimiento articular y test manual de fuerza muscular. Paidotribo.

De Ridder, R., Witvrouw, E., Dolphens, M., Roosen, P., \& Van Ginckel, A. (2017). Hip Strength as an Intrinsic Risk Factor for Lateral Ankle Sprains in Youth Soccer Players. American Journal of Sports Medicine, 45(2), 410-416. https://doi.org/10.1177/0363546516672650

Desmyttere, G., Gaudet, S., \& Begon, M. (2019). Test-retest reliability of a hip strength assessment system in varsity soccer players. Physical Therapy in Sport, 37, 138-143. https://doi.org/10.1016/J.PTSP.2019.03.013

Dierks, T., Manal, K., Hamill, J., \& Davis, I. (2008). Proximal and Distal Influences on Hip and Knee Kinematics in Runners With Patellofemoral Pain During a Prolonged Run. I Journal of Orthopaedic \& Sports Physical Therapy, 38(8), 448-456. https://doi.org/10.2519/jospt.2008.2490 
Ellison, J., Rose, S., \& Sahrmann, S. (1990). Patterns of hip rotation range of motion: a comparison between healthy subjects and patients with low back pain. Physical Therapy, 70(9), 537-541. https://doi.org/10.1093/ptj/70.9.537

Faul, F., Erdfelder, E., Lang, A., \& Buchner, A. (2007). G*Power 3: A flexible statistical power analysis program for the social, behavioral, and biomedical sciences. Behavior Research Methods, 39(2), 175-191.

https://doi.org/10.3758/BF03193146

Hafiz, E. (2014). The relationship of femoral torsion and lower limb injury. University of Sydney.

Hannon, J., Wang-Price, S., \& Garrison, J. (2019). Normalized hip and knee strength in two age groups of adolescent female soccer players. Journal of Strength and Conditioning Research, Epub ahead. https://doi.org/10.1519/JSC.0000000000003420

Hayen, A., Dennis, R., \& Finch, C. (2007). Determining the intraand inter-observer reliability of screening tools used in sports injury research. Journal of Science and Medicine in Sport, 10(4), 201-210.

Hides, J., \& Oostenbroek, T. (2016). The effect of low back pain on trunk muscle size/function and hip strength in elite football (soccer) players. Journal of Sports Sciences, 34(24), 23032311. https://doi.org/10.1080/02640414.2016.1221526

Hopkins, W. (2004). How to interpret changes in an athletic performance test. Sport Science, 8, 1-7.

Hopkins, W., Marshall, S., Batterham, A., \& Hanin, J. (2009). Progressive Statistics for Studies in Sports Medicine and Exercise Science. Medicine \& Science in Sports \& Exercise, 41(1),3-12. https://doi.org/10.1249/MSS.0b013e31818cb278

Howard, J., Fazio, M., Mattacola, C., Uhl, T., \& Jacobs, C. (2011). Structure, sex, and strength and knee and hip kinematics during landing. Journal of Athletic Training, 46(4), 376-385. https://doi.org/10.4085/1062-6050-46.4.376

Jackson, S., Cheng, M., Smith Jr, A., \& Kolber, M. (2017). Intrarater reliability of hand held dynamometry in measuring lower extremity isometric strength using a portable stabilization device. Musculoskeletal Science and Practice, 27, 137-141. https://doi.org/10.1016/j.math.2016.07.010

Kapandji, A. (2007). Fisiología Articular III: Miembro Inferior. Medica Panamericana.

Lange Chamorro, C. (2017). Confiabilidad y validez de un nuevo dinamómetro electromecánico funcional en la evaluación de la fuerza isométrica máxima de los rotadores mediales y laterales de hombro. Influencia de la posición articular. Universidad de Granada.

Lees, A., Asai, T., Andersen, T., ... H. N.-J. of sports, \& 2010, U. (2010). The biomechanics of kicking in soccer: A review. Journal of Sports Sciences, 28(8), 805-817. https://doi.org/10.1080/02640414.2010.481305

Leporace, G., Tannure, M., \& Zeitoune, G. (2018). Association between knee-to-hip flexion ratio during single-leg vertical landings, and strength and range of motion in professional soccer players. Sports Biomechanics, 19(3), 411-420. https://doi.org/10.1080/14763141.2018.1494207

Lindsay, D., Maitland, M., Lowe, R., \& Kane, T. (1992). Comparison of isokinetic internal and external hip rotation torques using different testing positions. Journal of Orthopaedic and Sports Physical Therapy, 16(1), 43-50. https://doi.org/10.2519/JOSPT.1992.16.1.43

Magalhães, E., Silva, A., Sacramento, S., Martin, R., \& Fukuda, T. (2013). Isometric strength ratios of the hip musculature in females with patellofemoral pain: a comparison to pain-free controls. The Journal of Strength \& Conditioning Research, 27(8), 2165-2170. https://doi.org/10.1519/JSC.0b013e318279793d
Meylan, C., Cronin, J., \& Nosaka, K. (2008). Isoinertial assessment of eccentric muscular strength. Strength \& Conditioning Journal, 30(2), 56-64. https://doi.org/10.1519/SSC.0b013e31816a7037

Ocarino, J., Resende, R., Bittencourt, N., Correa, R., Mendonça, L., Reis, G., Souza, T., \& Fonseca, S. (2021). Normative data for hip strength, flexibility and stiffness in male soccer athletes and effect of age and limb dominance. Physical Therapy in Sport, 47, 53-58. https://doi.org/10.1016/J.PTSP.2020.11.022

Palmer, M., \& Epler, M. (2002). Fundamentos de Las Técnicas de Evaluación Musculoesquelética. Paidotribo.

Paul, D., \& Nassis, G. (2015). Testing strength and power in soccer players: the application of conventional and traditional methods of assessment. The Journal of Strength \& Conditioning Research, 29(6), 1748-1758. https://doi.org/10.1519/JSC.0000000000000807

Powers, C. (2010). The Influence of Abnormal Hip Mechanics on Knee Injury: A Biomechanical Perspective. Journal of Orthopaedic \& Sports Physical Therapy, 40(2), 42-51. https://doi.org/10.2519/JOSPT.2010.3337

Romero-Franco, N., Jiménez-Reyes, P., \& Montaño-Munuera, J. (2017). Validity and reliability of a low-cost digital dynamometer for measuring isometric strength of lower limb. Journal of Sports Sciences, 35(22), 2179-2184. https://doi.org/10.1080/02640414.2016.1260152

Siff, M., \& Verkhoshansky, Y. (2004). Superentrenamiento (Segunda ed.). Paidotribo.

Sigward, S., Ota, S., \& Powers, C. (2008). Predictors of frontal plane knee excursion during a drop land in young female soccer players. Journal of Orthopaedic \& Sports Physical Therapy, 38(11), 661-667. https://doi.org/10.2519/jospt.2008.2695

Thorborg, K., Bandholm, T., Schick, M., Jensen, J., \& Hölmich, P. (2013). Hip strength assessment using handheld dynamometry is subject to intertester bias when testers are of different sex and strength. Scandinavian Journal of Medicine \& Science in Sports, 23(4), 487-493. https://doi.org/10.1111/J.1600-0838.2011.01405.X

Thorborg, K., Petersen, J., Magnusson, S., \& Hölmich, P. (2010). Clinical assessment of hip strength using a hand-held dynamometer is reliable. Scandinavian Journal of Medicine and Science in Sports, 20(3), 493-501. https://doi.org/10.1111/J.1600-0838.2009.00958.X

Vannatta, C., \& Kernozek, T. (2021). Normative measures of hip strength and relation to previous injury in collegiate crosscountry runners. Journal of Athletic Training. https://doi.org/10.4085/721-20

Vega Cerda, E. (2018). Validez y fiabilidad de diferentes protocolos de evaluación de la fuerza isométrica en la musculatura abductora de cadera con el uso de un dinamómetro. Granada: Universidad de Granada. http://hdl.handle.net/10481/54437

Widler, K., Glatthorn, J., Bizzini, M., \& Impellizzeri, F. (2009). Assessment of hip abductor muscle strength. A validity and reliability study. The Journal of Bone \& Joint Surgery, 91(11), 2666-2672. https://doi.org/10.2106/JBJS.H.01119

Wilczyński, B., Wąż, P., \& Zorena, K. (2021). Impact of Three Strengthening Exercises on Dynamic Knee Valgus and Balance with Poor Knee Control among Young Football Players: A Randomized Controlled Trial. Healthcare, 9(5), 558. https://doi.org/10.3390/HEALTHCARE9050558 